\title{
Apropiación de tecnologías de comunicación e información en el nivel de educación media superior. Transiciones en curso
}

\author{
Sagástegui Rodríguez, Diana \\ Apropiación de tecnologías de comunicación e información en el nivel de educación media superior. Transiciones \\ en curso \\ Revista Educación, vol. 42, núm. 2, 2018 \\ Universidad de Costa Rica, Costa Rica \\ Disponible en: http://www.redalyc.org/articulo.oa?id=44055139032
}

Esta obra está bajo una Licencia Creative Commons Atribución-NoComercial-SinDerivar 3.0 Internacional. 


\title{
Apropiación de tecnologías de comunicación e información en el nivel de educación
} media superior. Transiciones en curso

Appropriation of Information and Communication Technologies in High Schools: Ongoing Transitions

Diana Sagástegui Rodríguez

Redalyc: http://www.redalyc.org/articulo.oa?id=44055139032

Universidad de Guadalajara, México

dianasagastegui@yahoo.com

\section{Resumen:}

En la última década, diversos estudios internacionales han mostrado que los esfuerzos gubernamentales por dotar a las escuelas de tecnologías de información y comunicación (TIC) no han tenido los resultados esperados de mejora educativa. Para aportar en la comprensión de este fenómeno, se examinaron las formas de apropiación de tecnologías de información y comunicación (TIC) en escuelas públicas de nivel medio superior. Esta perspectiva analítica subraya el papel activo de estudiantes que ponen en juego un conjunto de intereses y significados al utilizar los dispositivos tecnológicos en situaciones específicas de sus contextos escolares, generando, frecuentemente, prácticas no previstas, distintas a las proyectadas (Andrés, 2014; Crovi, 2013; Laffey, 2004; Toboso-Martín 2013; Weber, Pisani y Francis, 2007). El estudio fue realizado en siete planteles escolares, mediante un acercamiento metodológico mixto, que incluyó aplicación de cuestionarios, observación participante y grupos de discusión. Los hallazgos muestran formas de apropiación caracterizadas por usos individualizados y procesos académicos rudimentarios en la consulta de información en línea y su referenciación, en un contexto escolar con acceso restringido a equipos de cómputo, deficiente conectividad en red, y ausencia de requerimientos pedagógicos en materia de documentación académica. Se discuten las implicaciones educativas de esta situación y se formulan cuestiones clave para enfrentar los desafíos que conllevan.

Palabras clave: Tecnologías de la información y la Comunicación, tecnología educacional, educación media superior.

\section{Abstract:}

In the last decade, several international studies have shown that governmental efforts to equip schools with information and communication technologies (ICT) have not had the expected results of educational improvement. To contribute to the understanding of this phenomenon, the forms of appropriation of information and communication technologies (ICT) in public high schools were examined. This analytical perspective emphasizes the active role of students who put into play a set of interests and meanings when using technological devices in specific situations of their school contexts, often generating unforeseen practices, different from those projected (Laffey, 2004; Weber, Pisani y Francis, 2007; Crovi, 2013; Toboso-Martín 2013; Andrés, 2014). The study was carried out in seven high schools, located in four cities of the state of Jalisco, with a mixed methodological procedure that included the application of questionnaires, participant observation sessions and discussion groups. It was identified that the appropriation of ICT among students is defined by individualized and pragmatic uses, aimed at speeding up the accomplishment of school tasks under simplification strategies that involve rudimentary academic processes, such as the consultation of online sources of information and their referencing. This situation occurs in an institutional context where there is limited access to equipment and network connectivity, and deficient school requirements in academic information management matters. The educational implications of this situation are discussed in order to face the challenges they entail.

KEYWORDS: Information and communication technology, Educational technology, Upper Secondary Education.

\section{INTRODUCCIÓN}

\author{
Los objetos no son solo entidades materiales. Son también realidades ideológicas, valores, \\ normas, culturas
}

Fuente: Jacques Lévy

El presente trabajo examina la apropiación de tecnologías de información y comunicación (TIC) en escuelas públicas de enseñanza media superior, a fin de discernir, a partir de los rasgos distintivos y las racionalidades con que estos recursos son utilizados, sus implicaciones educativas. A pesar de que estos 
dispositivos tecnológicos han sido adoptados prácticamente en todos los planteles escolares, no hay suficiente claridad sobre las repercusiones resultantes en materia de enseñanza y aprendizaje.

Existe una tensión irresuelta entre los discursos que conceden a las TIC un gran potencial para el diseño de nuevos modelos de enseñanza, aprendizaje y gestión de información (Banco Mundial 2016; OCDE 2010; 2012; 2015; UNESCO 2013; 2014) y los magros logros educativos escolares, de acuerdo con los resultados obtenidos en pruebas estandarizadas. El diagnóstico hecho con base en las evaluaciones realizadas en la primera década del siglo XXI por el Programa para la Evaluación Internacional de Alumnos (PISA) ${ }^{[2]}$, muestra la disociación existente entre la tasa de adopción de estas tecnologías y mejores niveles de aprendizaje. En América Latina, las escuelas han desempeñado en esta región un papel importante solamente en elevar el acceso de las poblaciones a las tecnologías digitales (Claro, Espejo, Jara y Trucco, 2011). Se infiere, entonces, que han contribuido a formar público consumidor de TIC, pero no necesariamente sujetos con mayores competencias.

Existen serias dificultades para que las TIC intervengan concertadamente a favor de los objetivos educativos institucionales, especialmente en impulsar el desempeño académico de estudiantes en las competencias en lectura, comunicación y matemáticas. En esta dirección, se reiteran las exhortaciones para remontar un estado de "déficit" en los sistemas escolares, y para subsanar la "brecha digital" que impide un uso "adecuado" de las tecnologías. Se insiste en asegurar el acceso de las TIC en las escuelas, diversificar los programas de software que allí se emplean, mejorar la formación de docentes y reconformar los procesos pedagógicos en el aula, que se mantienen fundamentalmente expositivos (UNESCO, 2015).

No es fácil distinguir la naturaleza de los aportes educativos de las TIC a la educación escolar, menos aún si se les trata de identificar de manera aislada. El reporte sobre estudiantes, computadoras y aprendizaje que realizó la Organización para la Cooperación y el Desarrollo Económicos (OCDE, 2012; 2015) [3] confirma lo anterior. En el caso específico de México, este estudio muestra que no hay evidencias de mejoras educativas en las escuelas atribuibles al factor tecnológico, y llega incluso a revelar que puede darse una correlación negativa: a mayor equipamiento tecnológico en las escuelas, menor grado de desempeño escolar de los estudiantes. El análisis destaca que, a pesar de que en México se utilizan más las computadoras para la enseñanza de las matemáticas que en el promedio de los países de la OCDE, los resultados de desempeño que obtiene el alumnado en esta área de conocimiento son más bajos. Contra lo que podría esperarse, en el área de matemáticas, estudiantes que utilizan más las computadoras, alcanzan menores competencias. Se hace hincapié en promover alfabetizaciones en cómputo y gestión de información, al igual que la formación en literacidades múltiples, para que el estudiantado desarrolle competencias comunicativas en plataformas tecnológicas (Lankshear y Knobel, 2010).

Sin demeritar la importancia de la enseñanza del uso de TIC, parece razonable empezar a analizar el rol educativo de las TIC en asociación con más factores, para lograr una comprensión más amplia sobre lo que está sucediendo. Por ejemplo, con base en un estudio comparativo en 2969 escuelas de 16 países de América Latina, Román y Murillo (2014) encuentran una correlación positiva entre el uso de computadoras y rendimiento académico en matemáticas y lectura en alumnado de sexto grado de primaria, con condiciones socioeconómicas y culturales similares, cuando cuentan además con docentes que, a su vez, las utilicen habitualmente. Se indica que contar con computadora en el hogar de estudiantes sí está asociado con mayores logros de aprendizaje, aunque este indicador se corresponde igualmente con un mayor nivel socioeconómico de escolares, variable que toma un mayor peso explicativo sobre los mejores resultados obtenidos.

En este tenor, la Comisión para la Educación de Calidad para Todos (2016) destaca que el impacto insuficiente del uso de TIC en las escuelas de América Latina está asociado a la corrupción que envuelve los programas de equipamiento y a que este se orienta prioritariamente a lograr indicadores políticamente rentables, tales como ampliar la cobertura educativa mediante modalidades de educación a distancia. Se pondera la necesidad de orquestar mejores estrategias, tales como promover el liderazgo de docentes para asegurar un mejor aprovechamiento de los -pocos o muchos- recursos tecnológicos disponibles, alcanzar una 
relación de 2 o 3 estudiantes por computadora, fomentar el aprendizaje colaborativo. Se recomienda que las inversiones económicas en TIC se hagan en función de logro de objetivos pedagógicos y no solo como una reacción a la oferta tecnológica.

Se reconoce la importante mediación que realizan las escuelas en los usos educativos de TIC, y se hace notar la necesidad de realizar investigación en esta dirección. Así, la $\operatorname{UNESCO}(2017$, p. 363) asegura que "la comprensión del impacto de las TIC en los resultados requerirá mejores datos sobre cómo, cuándo y cuánto los profesores y los alumnos utilizan las TIC”. Hacia este objetivo se orientó el presente estudio, con un interés centrado en la población de jóvenes estudiantes.

\section{Jóvenes, TIC y escuelas de bachillerato: Un estado de la cuestión en ciernes}

En México, la población joven, de 15 a 29 años, asciende a 30.6 millones, lo que constituye un 25,7\% del total de la población en el país. Aunque la educación media superior -también llamado bachillerato- es obligatoria, solamente un $32,9 \%$ de jóvenes ha alcanzado este nivel de escolaridad. En este ciclo educativo, la edad de la población estudiantil oscila mayoritariamente entre los 15 y los 21 años, grupo etario que es usuario familiarizado con el uso de TIC e internet.

Lejos de ser un grupo similar, el estudiantado de este nivel educativo es una población heterogénea de jóvenes que no caben fácilmente en estereotipos que les adscriben en "millenials", "generaciones google" "multimedia" o "Z", a quienes se les atribuye una capacidad innata para manejar con destreza cualquier tipo de dispositivo tecnológico. Por el contrario, la evaluación PISA de 2015 muestra que las competencias educativas en casi la mitad de estudiantes de 15 años -que cursaban el último año de secundaria o el primero de bachillerato- sin que existan diferencias entre mujeres y hombres-, no logran franquear el nivel más bajo de desempeño escolar; el 41,7\% tiene serias insuficiencias para realizar las tareas académicas más elementales en lectura, tales como identificar temas e ideas en un texto; un $47.8 \%$ tiene dificultad para distinguir y utilizar conocimientos científicos, y un 56.6 tiene dificultad para realizar operaciones y razonamientos matemáticos básicos (INEE, 2017).

Los resultados de la evaluación 2016 del Plan Nacional para la Evaluación de los Aprendizajes (PLANEA) en educación media superior en México (instrumento aplicado a escala nacional en abril de 2016 a 579923 alumnos en 14784 escuelas) son ilustrativos en este sentido: en el área de lenguaje y comunicación, el $71.1 \%$ se sitúa en un nivel de desempeño deficiente (45\% en nivel muy bajo, $26.1 \%$ en nivel bajo) y solamente el $9 \%$ se ubica en un nivel de desempeño alto. La tendencia en la competencia de lectura es a la baja, si consideramos que, en 2015 , el $64 \%$ se ubicaba en los dos niveles de logro inferiores (43,3\% en el nivel muy bajo y 20,7 en el bajo) (INEE, 2016).

Mucho se ha hablado de que el perfil de estudiantes en bachillerato refleja, en buena medida, el entono comunicativo en que vive la juventud. Se afirma que la generación actual de jóvenes ha vivido su vida habituada al visionado continuo de pantallas y se identifica al uso de TIC como distractor de sus estudios y se insiste en los usos riesgosos o problemáticos, en cuanto adicción, aislamiento, sexting, procrastinación (García-Gómez, 2017; Gomes-Franco y Sendín-Gutierrez, 2014; Sánchez Rodríguez, Ruiz-Palmero y Sánchez-Rivas, 2015). En contraparte, se valora la posibilidad de que las TIC hagan "más divertidas" o atractivas los procesos de enseñanza escolar, a riesgo del ahorro de atención y memoria, que conduce a una relación superficial con el conocimiento (Carr, 2011).

Se piensa que se lee menos debido a la ubicuidad de las pantallas. Pero más que un desplazamiento de los libros impresos por lecturas en pantallas, lo que parece ocurrir es una transición importante en la diversificación de los ritmos y los formatos de lectura y escritura en los grupos de jóvenes, que ya no corresponden solamente con los propios de la cultura letrada, sino con diversos lenguajes. En los soportes digitales se mezclan textos y lenguajes audiovisuales y se experimentan temporalidades más expeditas. Acontece una profunda ruptura que representa la lectura en la textualidad electrónica en relación con 
el orden de los discursos construida y sedimentada en siglos de modernidad. Hoy día, el libro, con un contenido específico y finito, un referente firme y estable, coexiste con otro tipo de lectura, discontinua, que paradójicamente resulta de la disolución de géneros, jerarquías y límites de las textualidades cuando se encuentran en plataformas digitales:

La lectura frente a la pantalla es generalmente discontinua, que busca, a partir de palabras claves o rúbricas temáticas, el fragmento textual del cual quiere apoderarse sin que necesariamente sea percibida la totalidad textual que contiene este fragmento. Así, en el mundo digital, todas las entidades textuales son como bancos de datos que procuran fragmentos, cuya lectura no supone la comprensión o la percepción de las obras en su identidad singular. (Chartier, 2018, p. 121)

Para este autor, no es la cantidad de lectura, sino un cambio en relación con lo escrito lo que está en curso. Y las transformaciones no solo derivan de la distinta materialidad de los soportes de lectura: se producen desplazamientos fundamentales entre lo público y lo privado, se diversifican los procesos socialización, se redefinen formas de inclusión y exclusión social. De ahí que el uso de artefactos tecnológicos no puede entenderse al margen de la cultura popular donde que se inscriben (Boyd, 2014; Cabero y Marín, 2014).

Para realizar sus prácticas mediadas por TIC, las generaciones jóvenes utilizan códigos y lógicas de ordenamiento distintos a los del texto impreso, más semejantes a las que adoptan las industrias culturales, que constituyen para ellas un referente principal y significativo en virtud de que la construcción de su identidad, sus prácticas de consumo y la construcción de subjetividad, procesos que se encuentran estrechamente vinculados entre sí (Dussel, 2010). De este modo, los grupos jóvenes no solo interactúan en contexto comunicativo altamente tecnologizado y mediático (Ferrés y Piscitelli, 2012), sino que es gracias a los intercambios comunicativos que pueden participar en un amplio repertorio de dinámicas económicas, sociales y culturales. Por esta razón, valoran las competencias de tipo pragmático y operacionales ágiles, que no son adquiridas dentro de las escuelas, sino en procesos de socialización distintos. En este contexto, no es la escuela la que les brinda las herramientas para desenvolverse en el mundo; ella está siendo colonizada por modalidades de acción externas, propias a entornos comunicativos de alcance global. Lo anterior no sucede sin dificultades, en la medida que el estudiantado se enfrenta a lógicas institucionales contrastantes (Weiss, 2012). Por una parte, las políticas relativas al uso escolar de TIC han tendido a ser conservadoras, focalizadas en ampliar el acceso a las tecnologías (Molino, 2017); por otro lado, los planes de estudio en la educación media superior mantienen un exceso de asignaturas y contenidos que favorecen la sobreinformación, la memorización de contenidos y la continuidad de métodos tradicionales de enseñanza y aprendizaje (Zepeda, 2017).

\section{Encuadre teórico: Apropiación de tecnologías}

Con lo visto hasta aquí, resulta evidente que el análisis de los usos de TIC en las escuelas es susceptible de estudiarse desde diversas perspectivas y encuadres teóricos, de muchas maneras. Una opción consiste en enfocarse a identificar coincidencias y desfases existentes entre un uso ideal y un uso real, entre las expectativas de manejo y los beneficios obtenidos, a la manera de varios estudios que se han citado más arriba.

Otros enfoques pueden interesarse en la adopción de tecnologías y, desde teorías de elección racional, descubrir las razones por las que se realizan determinados usos; a partir del análisis de variables como el género, la edad e intereses, se identifican los recursos que el público usuario pone en juego para lograr maximizar los dividendos obtenidos (Brown, Venkatesh y Hoele 2015; Özsungur y Hazer, 2018; Venkatesh y Ramesh, 2006).

Otras perspectivas toman distancia de la centralidad en la tecnología que subyace a los encuadres como los arriba descritos para colocar la cultura como un componente relevante en cualquier explicación de los vínculos entre las personas y objetos tecnológicos. El acento se coloca tanto en los factores contextuales y estructurales donde ocurren los usos de las tecnologías, como en la interpretación que hacen los sujetos 
de los artefactos, quienes, más que adoptar, adaptan las tecnologías en función de posibilidades, intereses y necesidades, pero también de consideraciones culturales y simbólicas.

Desde esta mirada, los usos de las tecnologías son considerados una construcción social dinámica. Al respecto, De Certeau (1990) advertía que, en cualquier práctica ordinaria, los sujetos no son consumidores pasivos de dispositivos tecnológicos, sino agentes activos que despliegan su agencia precisamente mediante las formas habituales que adquieren sus prácticas cotidianas. Por esta razón, los productos impuestos por un orden económico no son sometidos a un uso idéntico, sino a procesos de apropiación y reapropiación continua, a través de operaciones y manipulaciones diversas, que configuran racionalidades y formas de empleo de las tecnologías que no son generalizables, sino distintivas de sus contextos, y que se consideran acertados o desatinados desde criterios, principios y normativas del entramado cultural en donde se utilizan.

Pantzar (1997) argumenta que para comprender cómo las tecnologías son incorporadas a nuestras vidas cotidianas, es necesario un análisis social que dé cuenta de las dinámicas con las que son históricamente "domesticadas", esto es, integradas en los rituales cotidianos, a lo largo del tiempo. La domesticación implica la integración de un objeto técnico en un sistema más amplio de objetos, lo que provoca que adquieran expresiones y usos que muchas veces no fueron imaginados por quienes los diseñaron. Piénsese, por ejemplo, en los usos iniciales a los que estaban destinados el automóvil o el teléfono.

En nuestro trabajo, optamos por un marco de apropiación de tecnologías que, con ciertas variaciones, tiene una larga trayectoria en el campo de los medios y la comunicación (Morales y Loyola, 2009). La apropiación es comprender un conjunto de encuadres teóricos, cuyo común denominador es el interés por analizar la articulación que se produce entre una determinada oferta tecnológica, por un lado, y el marco de valores y significaciones sociales por otro, lo que da como resultado determinadas modalidades de uso (Andrés, 2014; Cabello y López, 2017; Laffey, 2004; Crovi, 2013; Toboso-Martín 2013; Weber, Pisani y Francis, 2007). En ningún caso se puede sostener que existe un uso "adecuado" de las TIC derivado exclusivamente del aprovechamiento de las capacidades tecnológicas de los artefactos; es necesario contemplar dinámicas y factores contextuales, de orden social, económico y cultural. En este encuadre teórico, desarrollamos nuestro estudio sobre la apropiación de las tecnologías en contextos escolares, con la finalidad de analizar sus implicaciones educativas.

\section{Metodología}

Para la realización de este estudio, se diseñó una muestra no probabilística que consistió en siete escuelas públicas de bachillerato de modalidad presencial, en dos subsistemas de educación pública. A fin de contar con una base comparativa entre poblaciones de distintos estratos socioeconómicos, en diferentes áreas urbanas y desiguales índices de marginación social, las escuelas se seleccionaron de la siguiente manera:

a) Cuatro escuelas emplazadas en la ciudad de Guadalajara, capital del Estado de Jalisco, dos de ellas situadas en zonas urbanas cuya población pertenece a un estrato socioeconómico medio; las otras dos situadas en zonas desfavorecidas, donde habitan poblaciones de bajos ingresos.

b) Tres escuelas ubicadas en ciudades del mismo Estado de Jalisco, no mayores a 15 mil habitantes; dos planteles ubicados en municipios de marginación social baja y una escuela localizada en un municipio de marginación social media ${ }^{[4]}$.

Se obtuvo información mediante un procedimiento metodológico mixto; datos cuantitativos se obtuvieron mediante un cuestionario aplicado a grupos de $5^{\circ}$ y $6^{\circ}$ semestres (los últimos del ciclo escolar) ${ }^{[5]}$; se interrogó sobre las condiciones de acceso y los usos de TIC para prácticas y trabajos escolares, ya sea dentro o fuera de las aulas. Se aplicaron 1102 cuestionarios en 2011 y 1104 cuestionarios en 2014; en total 2 206, de los cuales 1191 fueron contestados por mujeres y 1015 por hombres, cuyas edades se encuentran 
en un rango entre 16 años la edad mínima y 25 la edad máxima, siendo la media 18 años y la moda 16 años. No se encontraron diferencias significativas en los datos desagregados por género, por lo que los resultados se presentan agregados.

Se llevaron a cabo adicionalmente otros dos procedimientos con la finalidad de obtener datos cualitativos, para fines de triangulación e interpretación: cuatro grupos de discusión y siete sesiones de observación participante en el mismo número de bibliotecas escolares, en periodos de tiempo de aproximadamente 40 minutos cada una.

\section{Resultados}

\section{Condiciones de acceso y características de uso de TIC en las escuelas}

Aunque las condiciones de acceso y características de uso de las TIC en las escuelas se mantienen en continua redefinición, algunas tendencias han adquirido ya cierta estabilidad. Encontramos que el acceso a estos recursos sigue siendo desigual; se registraron diferencias significativas asociadas a la ubicación espacial de las escuelas. Tal como se esperaba, encontramos una correlación positiva entre grado y calidad del equipamiento y el nivel socioeconómico del alumnado, determinado por el sitio donde se ubica la escuela. En contraste, no se detectaron diferencias significativas en cuanto a cómo son empleadas las TIC para procesos educativos escolares, ni entre las escuelas en estudio, ni por el género de estudiantes.

Esta similitud indica un poder de mediación importante de la escuela para modelar las prácticas educativas, independientemente de la disponibilidad y características de los recursos tecnológicos. No es sorprendente; constatamos que la disposición espacial de las TIC en las escuelas y los usos a las que están destinadas es muy homogénea. Las computadoras no se emplean al interior de las aulas, sino principalmente en las salas de cómputo y biblioteca, mientras que estudiantes las emplean en sus hogares para hacer trabajos encargados por sus docentes. De allí que, como se mostrará más adelante, las prácticas de enseñanza y aprendizaje en las escuelas no se han visto especialmente redefinidas por el uso de tecnologías.

En las escuelas opera una suerte de sistema normativo que organiza la disposición espacial de las TIC. En las aulas, generalmente no se encuentran computadoras, con excepción de presentaciones de contenidos que se proyectan algunas veces durante las clases. Como resultado, las aulas siguen siendo el espacio destinado a las disertaciones magisteriales y mantiene al resto de los espacios escolares como satélites que giran en torno a la enseñanza en ellas.

Las computadoras se ubican en salas o aulas de cómputo y son dedicadas principalmente a la búsqueda de información, la elaboración de trabajos y otra serie de actividades puntuales, como ejercicios didácticos en algunas asignaturas o responder las pruebas estandarizadas. Como se mencionó, las computadoras también se encuentran en las bibliotecas escolares, donde son utilizadas fundamentalmente para hacer búsquedas de información y, en menor medida, para realizar tareas y trabajos.

Si el espacio no es solamente un escenario o trasfondo, sino la expresión de ordenamientos sociales (Lévy, 2010), a esta disposición espacial subyace sin duda una concepción de educación, de sistema escolar y una visión sobre las funciones para las que pueden ser útiles las TIC. Al respecto, puede señalarse que la incorporación de TIC no ha representado cambios en la tradicional disposición espacial de las escuelas, ni en la división del trabajo escolar que favorecen. Al estar el uso de TIC confinado en espacios fuera de las aulas, denota una racionalidad que configura ciertas formas de apropiación, e inhibe otras. Por ejemplo, no favorece que los usos de TIC sean guiados por sus docentes, ya sea explorar estrategias de construcción conocimientos en procesos colaborativos, diversificar fuentes de información y representación de conceptos, explorar el desarrollo conceptual con recursos visuales ampliados, y, en general, estimular la participación de estudiantes durante los tiempos de clases (Romero y Quesada, 2014). Quedan fuera también las posibilidades de diseñar 
entornos de aprendizaje personalizados, estrategias de trabajo grupales o plataformas colaborativas en red, idealmente planteadas como vías de contrastación entre varias fuentes de información, diferentes puntos de vista. Esta larga lista de usos que son desalentados constituyen estrategias diseñadas para romper -al menos en cierto grado- la propensión a la docencia expositiva, que tiende a encauzar el aprendizaje como una actividad centrada en obtener información puntual y homogénea sobre un tema preestablecido en una asignatura del currículo escolar.

Otras implicaciones educativas pueden ser inferidas de esta situación; atañen a las competencias del alumnado en materia de gestión de información y de conocimientos. Para realizar sus trabajos escolares, los grupos de estudiantes no consultan recursos de información académica accesibles en red, como canales educativos, blogs científicos, sitios de universidades, asociaciones o entidades públicas dedicadas a la comunicación pública de la ciencia, e organizaciones e institutos de investigación, ni acervos académicos de acceso abierto. Mientras tanto, en las aulas se refuerzan paradigmas y recursos de aprendizaje tradicionales: los estudiantes utilizan libros y cuadernos. Los apuntes escolares que se realizan en clase, a partir de la exposición -ya sea del profesorado o de otros estudiantes- son un insumo muy recurrido.

La multiplicidad de funciones que con una computadora se pueden realizar de manera simultánea determina, en cierto grado, su forma de uso; el estudiantado manifestó que, al realizar sus tareas escolares con ayuda de la computadora, atienden varias cosas a la vez: contestan correos, "chatean", postean en redes, escuchan música. Es así como integran las actividades orientadas al aprendizaje en flujos de actividad y de manejo de información más amplios. Puede inferirse por estos datos que las lecturas son muy probablemente de textos cortos y fragmentados, lo que no parece entorpecer el logro de sus objetivos escolares cotidianos. El estudiantado distingue entre materiales impresos y digitales, y le atribuyen alcances desiguales por los grados de atención que exigen y el aprendizaje que logran con su lectura. La totalidad de escolares, sin excepción, conviene en que, cuando necesitan asegurar el logro de un aprendizaje determinado -por ejemplo, para preparar un examen- prefiere leer en soportes impresos y se concentra solamente en ellos, sin otras actividades ni distracciones. En este sentido, sí identifican desiguales procesos de aprendizaje de acuerdo con los dispositivos tecnológicos utilizados, que pueden estar relacionados con los procesos cognoscitivos involucrados, pero no es posible saberlo en nuestro estudio. ${ }^{[6]}$

En lo que atañe a las bibliotecas escolares, libros y TIC también son claramente diferenciados, en diferentes modos. Las bibliotecas disponen de computadoras para el uso estudiantil -en un número de que puede ir de las dos a las doce por plantel educativo-, equipo que se les facilita solo por tiempo limitado y exclusivamente para realizar tareas escolares; está especialmente prohibido el acceso a las redes sociales. Llama la atención la escasa capacidad de agencia que le es acordada a estudiantes también en la biblioteca. Así como en el aula, el personal docente sigue manteniendo la pauta de las actividades, en las bibliotecas, el personal encargado sigue concentrando el poder de la búsqueda de los acervos físicos. A pesar de que los acervos de las bibliotecas escolares están digitalizados, los grupos de estudiantes deben pedir ayuda al personal bibliotecario para el acceso y préstamo de materiales impresos.

En contraparte, las computadoras destinadas a la utilización de estudiantes son para usos puntuales; no están destinadas al desarrollo de competencias en materia de búsqueda de información en internet, ya sea en bases de datos académicos, canales de video educativos, o en bibliotecas virtuales a las que se puede tener acceso. Tampoco se promueve en la biblioteca el uso de recursos de acceso abierto para búsquedas de información académica, ni se enseña el amplio repertorio de aplicaciones personalizadas que permiten organizar el inmenso universo de información disponible en internet, entre las que se pueden mencionar las alertas automáticas y los marcadores sociales.

El personal de bibliotecas no parece estar interesado en ayudar al desarrollo de capacidades para la creación de contextos y recursos dinámicos de aprendizaje, ni en propiciar en estudiantes competencias que son esenciales a fomentar el uso competente y ético de los recursos de información disponibles (Tripp, 2011) lo que resulta especialmente importante cuando la copia encubierta de textos parece convertirse en moneda 
corriente en los trabajos escolares, lo que, a su vez, ha derivado en el auge de programas de software destinados a la detección de plagio.

Por lo demás, cabe preguntarse qué tan vigente puede ser mantener en los tiempos actuales el mismo patrón de las bibliotecas escolares en el bachillerato, originalmente dedicadas a ofrecer solo recursos de información sobre los contenidos establecidos por las asignaturas del plan de estudios. La costumbre de crear una suerte de "cerco informativo" se ha heredado a la biblioteca digital, recurso con que cuenta uno de los sistemas de educación media superior estudiados ${ }^{[7]}$ : Consiste en un acervo de textos digitalizados, disponibles en línea, consagrado exclusivamente a ofrecer materiales para los contenidos de las asignaturas del plan de estudios. Semejante modelo resulta todavía menos funcional; no es apreciado por parte del alumnado, quienes declararon en los grupos de discusión que no se valen de este recurso, dado que encuentran más práctico hacer la búsqueda de la información directamente en internet, a través de un buscador como Google.

Este ordenamiento espacial de los equipos y funciones crea, además, desequilibrios en el aprovechamiento de recursos tecnológicos, pues mientras las salas o "aulas de cómputo" pueden estar sin ser utilizadas por periodos de tiempo relativamente largos, las computadoras de la biblioteca siempre son insuficientes: tienen mucha demanda por parte de estudiantes, pues son las que están disponibles para revisar sus cuentas de correo y hacer tareas escolares. Y por otra parte, el uso del equipamiento tecnológico se mantiene en un modelo de "servicio" que se presta al alumnado. Pero la disposición espacial de TIC disponible en salas de cómputo y en bibliotecas sirve para administrar la escasez de equipamiento, que se agrava en escuelas con poblaciones más vulnerables y económicamente más desfavorecidas; se encontró un caso donde la biblioteca escolar no contaba con personal bibliotecario por lo que era "atendida" por personal distinto al de esa área.

Es especialmente deplorable que las políticas educativas consideren como indicador de "acceso" cuando se cuenta con una computadora por escuela:

El desarrollo de habilidades digitales, específicamente el uso de internet, es un derecho humano reconocido en el artículo $6^{\circ}$
constitucional que garantiza que toda persona puede acceder a las Tecnologías de la Información y la Comunicación (TIC),
así como a los servicios de banda ancha e internet (Naciones Unidas, 29 de junio de 2012; Decreto, DOF, 11 de junio de
2013). En el Sistema Educativo Nacional (SEN) se enfatiza el desarrollo de dichas habilidades, ya que se presentan como un
eje transversal en el currículo nacional. Por lo tanto, sería necesario o deseable que las escuelas contaran con al menos una
computadora para uso educativo y con servicio de internet. (INEE, 2017)

Sería más necesario y deseable que hubiera más; en el año 2013, solamente el 42,9\% de las escuelas de nivel medio superior en México contaban al menos con una computadora por cada ocho estudiantes, y solo el 44.2\% contaba al menos una computadora con conexión a Internet (INEE, 2014). Considérese lo que esto significa en un país donde -con datos de 2014-, el 53,9\% de la población menor a 18 años está en situación de pobreza ${ }^{[8]}$, que no les permite solventar, por su cuenta, la compra de una computadora personal, y tienen fuertes limitaciones para pagar la conexión a ,internet desde sus dispositivos móviles -principalmente los teléfonos inteligentes-.

Como puede advertirse, son variadas las maneras comolas escuelas tienden a apuntalar la desigualdad social y educativa. Los sistemas educativos públicos se han mantenido en una posición confortable, declarándose de manera impropia como escuelas "conectadas", cuando el alumnado y profesorado que pueden usar internet en bibliotecas y aulas de cómputo son una proporción relativamente pequeña. No resulta, entonces, sorprendente que los datos obtenidos muestran además que decrece la importancia de las escuelas como sitio de conexión a internet: mientras que en 2011, el 64\% de alumnado declaró conectarse a internet desde su casa, y un 53\% afirmaba hacerlo también en la escuela, para 2014, un 85,9\% reportó conectarse a internet desde su hogar, en tanto el porcentaje que declaró conectarse también en la escuela bajó hasta un 43,9\%, como se muestra en la Figura 1: 


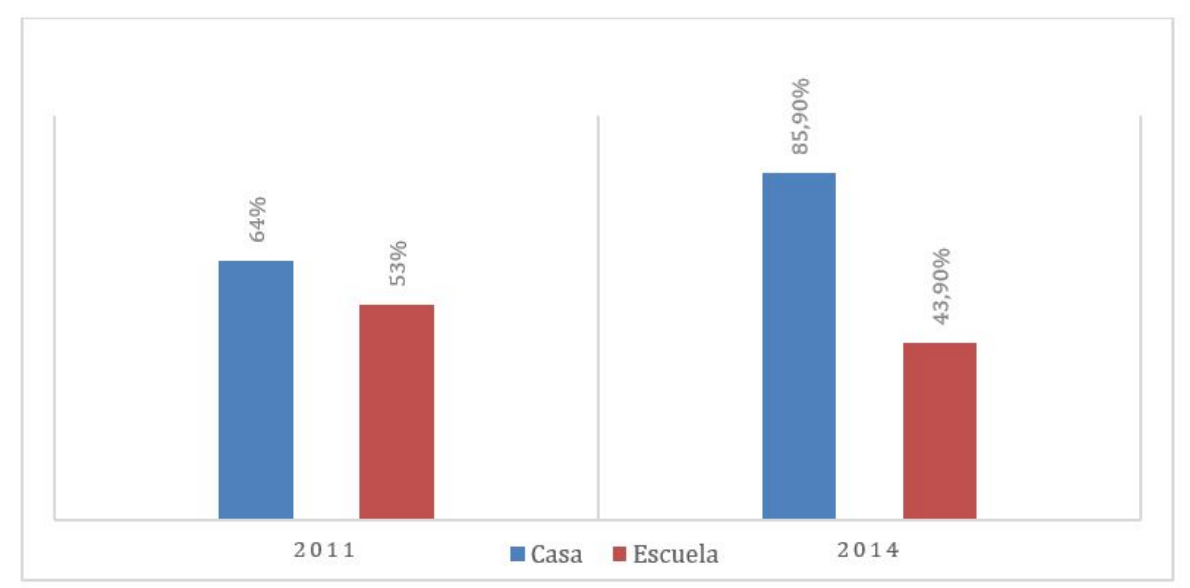

FIGURA 1

Sitios de acceso a internet de estudiantes

Nota: En esta figura y las cuatro subsiguientes, cuando se establecen porcentajes nos referimos a las respuestas obtenidas en los cuestionarios, aplicados en 2011 y 2014 . Nótese que, en algunas ocasiones, las respuestas en las diferentes opciones pueden contabilizar más de un $100 \%$, dado que podían señalar en sus respuestas varias opciones que no eran necesariamente excluyentes.

La conectividad a internet se ha incrementado notablemente entre estudiantes en este lapso de tiempo, pues mientras en 2011 solamente $24,81 \%$ se conectaba de tres a cinco horas al día entre semana, en 2014 este porcentaje asciende a $73,7 \%$. Resulta difícil identificar para qué se conectan, o qué recursos de la red aprovechan para propósitos educativos. Sobre este aspecto, puede señalarse como indicativo la baja proporción de estudiantes que domina otra lengua: en 2011 el 84\% no había leído nunca una página web en otro idioma; en 2014, afortunadamente el porcentaje desciende al 77.3\%, lo que de cualquier modo sigue siendo una alta proporción que se ve restringida a consultar sitios exclusivamente en idioma castellano, lo que limita considerablemente sus posibilidades de uso con fines académicos.

Otras restricciones operan también. Por ejemplo, cuando ciertas formas de utilización de TIC son desestimadas en las escuelas, a pesar de ser más extendidas. Considérese que, en 2014, un 70,6\% del alumnado refirió el teléfono inteligente como la forma de conexión principal a la red. No obstante, el uso de estos artefactos se prohíbe en las aulas por considerarse ajeno a la actividad educativa de las escuelas. Ciertamente, los usos de los teléfonos inteligentes por parte de estudiantes no están dirigidos a fines académicos, pero lo mismo puede decirse de otros dispositivos como las computadoras portátiles y tabletas que, en los casos en que cuentan con ellas, no se prohíbe su uso, aunque tampoco se promueve dentro de las aulas. Para mucho profesorado, las TIC en los salones de clase no hacen más que "distraer" la atención de sus estudiantes.

$\mathrm{Al}$ respecto, conviene reflexionar en qué medida se puede sostener una distinción categórica entre usos educativos y de otro tipo. Como se mencionó más arriba, los grupos de jóvenes utilizan las tecnologías con diversas aplicaciones al mismo tiempo y son a la vez una mediación para participar en la cultura popular, de manera bastante semejante, aunque también creativa: en 2011, escolares expresaron que los materiales que bajan de la red son principalmente música (88\%), fotografías (78\%) y videos (63\%); un patrón similar se mantiene en 2014, correspondiendo un $87,4 \%, 77,7 \%$ y $68,1 \%$ para música, fotografías y videos respectivamente. Este espectro de uso tiende a ampliarse a lo largo del tiempo: nótese el 43,3\% de alumnado que declara en 2014 haber descargado artículos académicos, tipo de material que no apareció en las respuestas de los cuestionarios en 2011, como puede apreciarse en la Figura 2. 


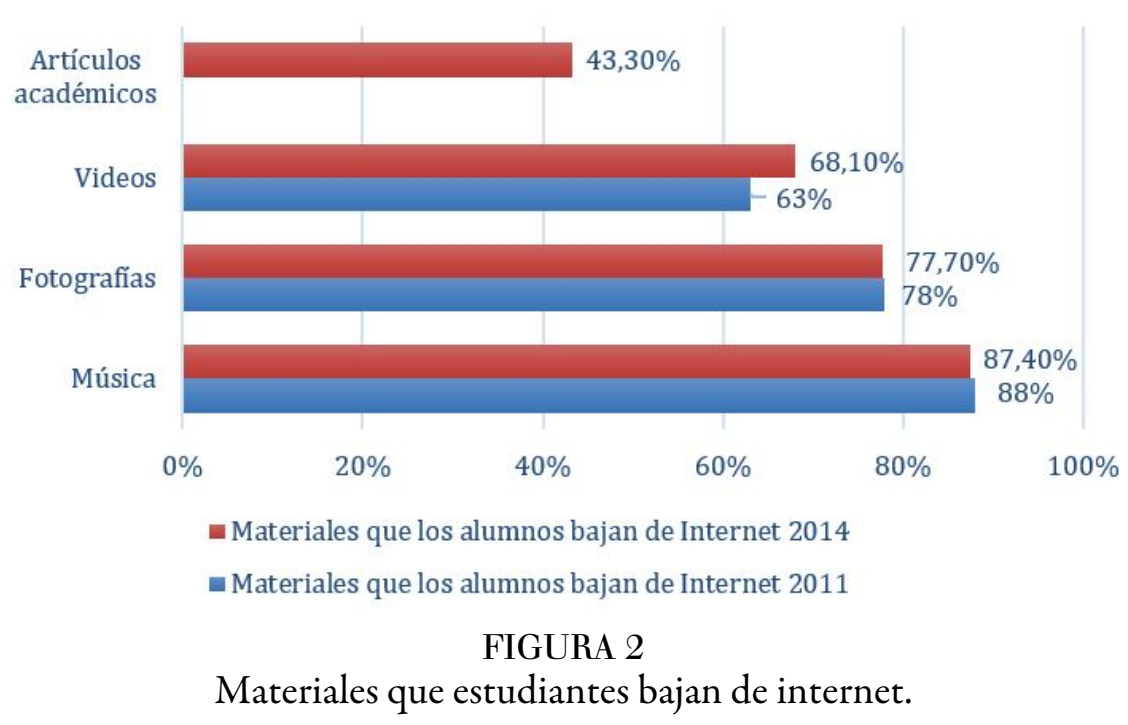

Conviene aquí señalar la convergencia entre los contenidos de la cultura popular y otros géneros y lenguajes, fenómeno reforzado a su vez por la digitalización de la información que posibilitan las TIC. Una resultante de esto es el rol de estudiantes como "productores o productoras de contenidos", que se mantiene, sin embargo, con un estándar estrecho, modelado principalmente por la oferta comercial. En 2011 la mayor proporción de materiales que habían subido a internet fueron fotografías en redes sociales en un 75\% (que remonta a un $81,8 \%$ en 2014). La colocación de videos en redes sociales es una práctica que pasó de conformar un $39,4 \%$ en 2011 a un $47,1 \%$ en 2014, como se observa en el la Figura 3.

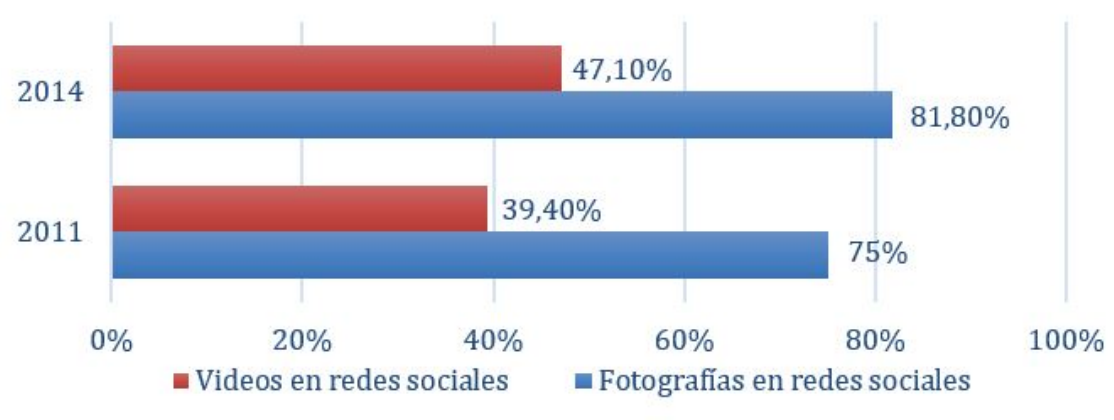

FIGURA 3

Materiales que estudiantes suben a internet

La tendencia a privilegiar los usos recreativos, lúdicos y de socialización se sostuvo en ascenso a lo largo del tiempo: la participación en las redes sociales se incrementa notablemente, especialmente en Facebook, cuya participación es considerada por el alumnado como una fuente de información útil para sentirse integrado en las dinámicas de sus grupos sociales de referencia, pero también para hacer circular diversos tipos de información, incluida aquella relacionada con las tareas escolares de estudiantes. En 2011, un 82,4\% de estudiantes afirmó utilizar Facebook., en tanto YouTube era utilizado por el 89,2\%, mientras solo el 18,6\% había utilizado Twitter. Estos datos se corresponden con los obtenidos en 2014: el 94,5\% se declara público usuario de Facebook, y 93,9\% de YouTube. Un 30,7\% dice usar Twitter, si bien solo el 7\% dice usarlo diariamente, en contraste con un $63,1 \%$ que afirma consultar diariamente Facebook. En este mismo año, un 28,7\% mencionó utilizar WhatsApp, aplicación que no estaba disponible aún en 2011.

Las redes sociales constituyen una expansión sin precedentes no solo en cuanto a fuentes de información, sino en la mezcla de géneros, medios y formatos de comunicación que, sin duda, pueden ser aprovechables 
para actividades escolares de tipo colaborativo y para expresión de un sinnúmero de temas académicos, como se ha mostrado en diversos estudios. Sin embargo, el uso de estas plataformas se resiste a generalizaciones fáciles y sigue siendo controvertido; Chen y Bryer (2012) encontraron que la utilización de las redes sociales para fines educativos no puede seguir un canon único, pues depende, en gran medida, de procesos experimentales; pero representa, sin duda, la posibilidad de acceder a una mayor información, flexibilidad, participación y conectividad entre estudiantes, terreno fértil para logro de aprendizajes. No hay que perder de vista que las redes sociales son entornos complejos, que no se reducen a simples facilitadoras de participación e intercambio: son parte de consorcios comerciales, dedicados a la mercantilización a partir de la construcción de identidades, consumos y construcción de significado, lo que constituye un conjunto de serios desafíos de discernimiento intelectual y ético (Rodríguez-Hoyos; Haya- Salmón y Fernández-Díaz, 2015).

Estamos frente a una compleja estructura comunicativa y de sociabilidad que, sin embargo, también es susceptible de someterse al servicio de prácticas docentes tradicionales; así lo advierten Manca y Ranieritt (2016), quienes, a partir de una extensa revisión del estado del arte de investigaciones sobre el potencial de las redes sociales como Facebook, coinciden en que, si bien esta red social representa un entorno social dinámico y flexible, generalmente las personas jóvenes toman ventaja de la popularidad y el aura de entretenimiento que esta red social representa, mientras sus potencialidades educativas, si fuera el caso, resultan omitidas.

En una crítica aún más punzante, Van Dick (2016) señala que estas plataformas son sistemas automatizados que, desde bastiones empresariales cuasi monopólicos, impulsados por sus propias agendas, que no son propiamente educativas, integran publicidad, causan desinformación intencionada, inducen adicción a la conectividad y sustraen de forma subrepticia datos de los sujetos usuarios con fines de lucro. Esta autora advierte, además, que su funcionamiento a través de algoritmos no solo vehicula y facilita los intercambios, sino que los programa, en vista de lo cual, la labor realmente educativa estaría orientada a desentrañar las lógicas que operan estos ecosistemas comunicativos.

\section{Prácticas no previstas y otros desplazamientos en la cultura escolar}

Hoy en día, TIC e internet forman parte ineludible de la vida de estudiantes; quienes, para la realización de trabajos escolares desarrollan ciertas habilidades en el acceso y el manejo de información en red que se han hecho funcionales y útiles para las exigencias y usanzas propias de la cultura escolar. Asistimos a transiciones fundamentales en cuanto al acceso y manejo de información, en un entorno de ubicuidad y abundancia, e igualmente de dispersión. Importantes formas de apropiación de TIC en las escuelas se hacen visibles cuando el alumnado indica cómo procede ante el inmenso caudal de datos que se encuentra disponible en línea. Y aquí, nuevamente, los profesores y profesoras marcan la pauta.

Los grupos de estudiantes señalan que, para tomar decisiones sobre qué material seleccionar, se basan en la información previa que les ha dado su docente sobre un tema. Para valorar y elegir lo información adicional que necesitan para hacer sus trabajos y tareas escolares, se apoyan, también, en los apuntes que hacen en clase. Con esta base, deciden qué información conviene tomar de internet, a partir de su cotejo. Declaran también otra forma habitual de proceder para este objetivo; consiste en comparar la información que aparece en dos o tres sitios web; si no hay divergencias, entonces eligen cualquiera de ellos, porque estiman que hay buena probabilidad de que la información sea confiable. Nadie aludió ni consideró digno de comentarse el sistema algorítmico que determina el orden de la aparición de la información que se busca en línea con un motor de búsqueda.

Como se hizo notar más arriba, para la realización de sus trabajos escolares, Google es un recurso protagónico e indispensable. Pero su rol ha sufrido a su vez transiciones a lo largo del tiempo. En el cuestionario aplicado en 2011, un 57,2\% de estudiantes mencionó que buscan la información que necesitan para hacer sus tareas escolares a través de este robot. Se constata que, en el entorno de red, las revistas electrónicas especializadas no constituyen para ellos fuentes de información a donde recurran, excepto para 
un 5\%. En lo que toca a fuentes de información impresos, los grupos escolares mencionan los libros de texto, que afirman consultarlos habitualmente solo un $24,8 \%$.

Sorprendentemente, Google se emplea menos en 2014: la proporción de alumnado que emplea este buscador para realizar sus trabajos escolares baja a un 28\%; correlativamente, quienes declaran usar sus apuntes de clase también es un porcentaje menor, que suma apenas el 25,1\%; los libros de texto también resultan ser menos solicitados: son fuente de información para un escaso 18,6\%. Pero en 2014, aparece otra fuente de consulta: un 46,8\% acude a sitios web donde se encuentran repositorios especialmente dispuestos para facilitar la realización de tareas escolares. El comparativo y los cambios pueden apreciarse en la Figura 4.

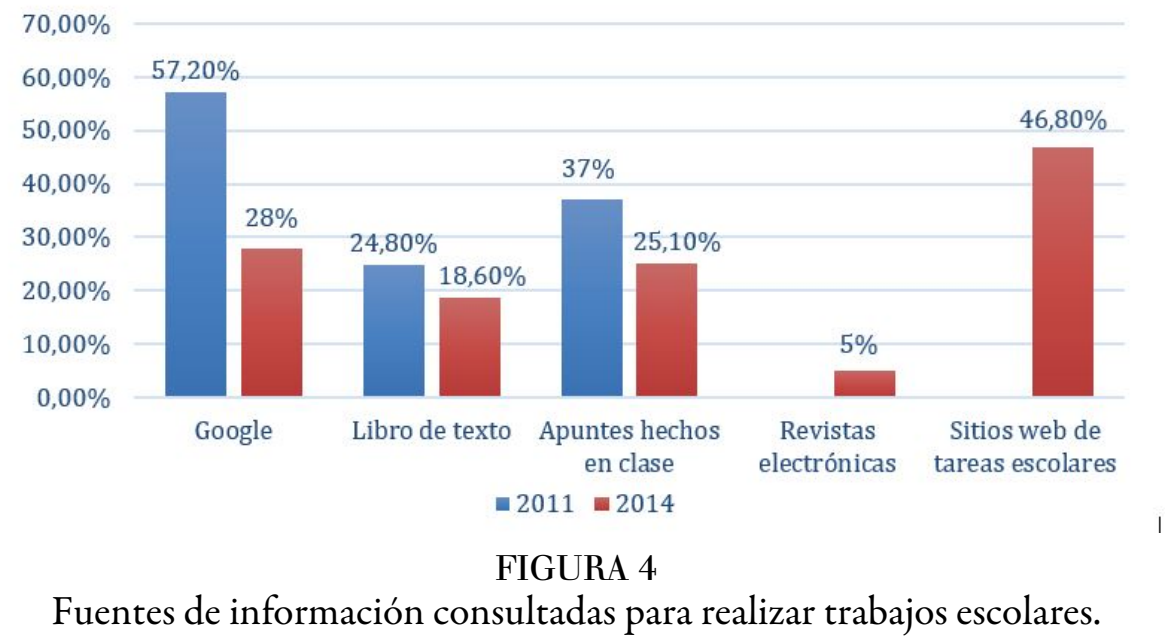

En un lapso de apenas tres años, el estudiantado ha variado de manera importante sus formas de gestión de información en línea, en lo que corresponde a las fuentes de información utilizadas. Relacionado con lo anterior, encontramos que su percepción valorativa acerca de la abundancia de datos en la red también se modificó, de manera no prevista: si en 2011, un 89,4\% manifestó que poder acceder a una gran cantidad de información a través de internet era una ventaja para su vida escolar, a la vez que un 57,3\% percibía que este exceso de información es igualmente una desventaja, por la dificultad que representaba discriminar y evaluar su calidad, encontramos que para el año 2014, solo el 25,4\% refirió como factor ventajoso la extensa cantidad de información que existe disponible en internet; también disminuyó el porcentaje que lo considera una desventaja hasta un 40,4\%, como se muestra en la Figura 5.

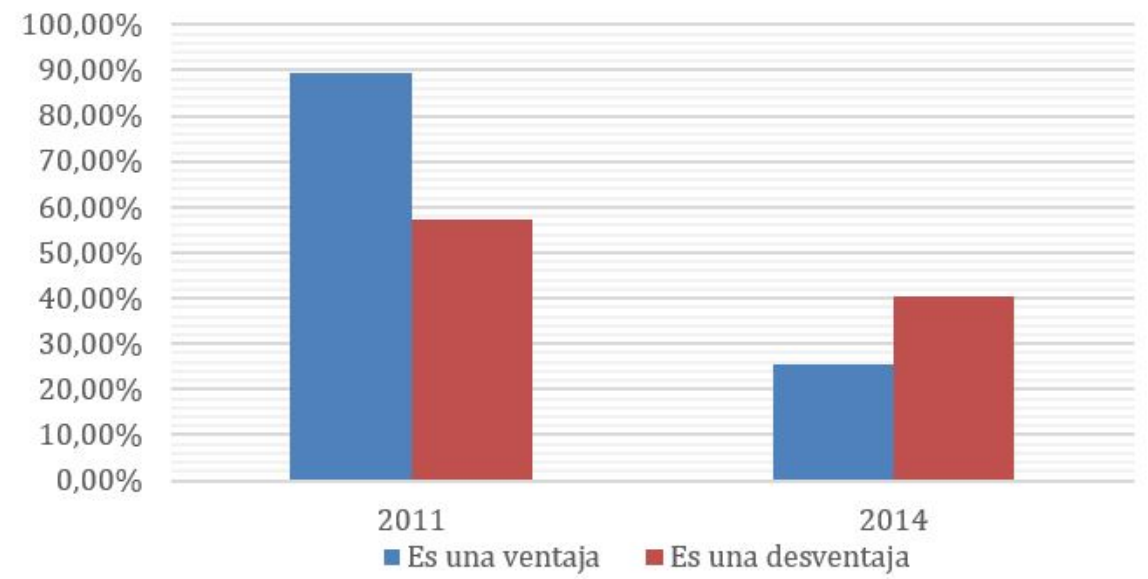

FIGURA 5

Valoración de estudiantes sobre la amplia cantidad de información disponible en internet. 
Estas diferencias encontradas entre 2011 y 2014 pueden explicarse, al menos en parte, por la emergencia de otras fuentes de información: sitios web "especializados" en tareas escolares. El éxito de estos sitios reside en que logran producir un modelo textual que conjuga la diversidad y multiplicidad propias del acceso digital con la ordenación temática y localizada propia de los libros de texto escolares. Así, en 2014, los grupos de estudiantes mencionaron dos sitios de búsqueda de información que son una suerte de "repositorios" de tareas escolares. Un 46,8\% de estudiantes afirman consultarlos diariamente; se trata de El rincón del vago y Buenas tareas. Para ilustrar su naturaleza, sirva la autodefinición del sitio de internet Buenas tareas que postulaba en 2014:

Nuestra misión es proporcionar un conjunto cada vez mayor de herramientas de aprendizaje en línea para ayudar a los estudiantes a tener éxito. Esto lo hacemos con nuestra creciente biblioteca de casi 13 millón [sic] de notas de cursos, trabajos de investigación, ensayos, trabajos finales e informes de libros cargados por los estudiantes para todos tus dispositivos. También proporcionamos notas de AP que te ayudarán a ser una estrella en tus exámenes, y notas de libros para ayudar a explicar obras literarias famosas. ¿No sabes por dónde empezar? Comienza por la búsqueda de un tema específico o navega por nuestra extensa lista de categorías ${ }^{[9]}$.

También Buenas Tareas ha evolucionado y perfecciona sus estrategias narratológicas; para 2017, se presenta en forma ya no como apoyo instrumental para realizar con éxito trabajos y exámenes escolares, sino como un sitio para realizar investigación, con diversas opciones de utilización, incluyendo dispositivos digitales móviles, integrando además indicadores bibliométricos de impacto de las obras del acervo, como puede apreciarse en la Figura 6.

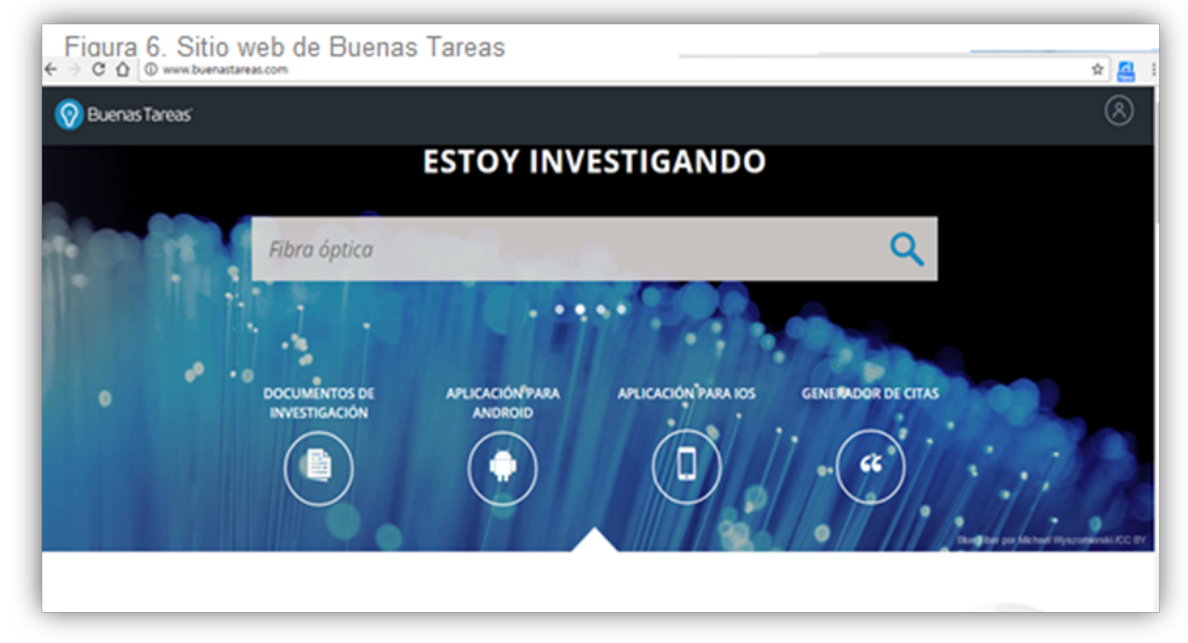

FIGURA 6

Sitio web Buenas Tareas

Nota: Tomado de http://www.buenastareas.com/. Consultado en enero de 2017.

Este sitio presumía contar con más de 34 millones de "miembros", más de once millones de documentos y 45 mil temas de ensayo. Se vanagloria de haber aparecido en medios de comunicación -como la cadena CNN, La Razón, Publímetro y Dinero en Imagen-. También se declara determinado a desalentar la deshonestidad académica y cualquier uso indebido de la información que ofrece:

Para prevenir y desalentar la deshonestidad académica, trabajamos con servicios como Turnitin para rastrear nuestro contenido e incluirlo en sus bases de datos. Nunca presentes ningún material de este sitio web como tuyo y siempre incluye las citas adecuadas. Las citas se pueden encontrar al final de cada uno de los documentos en BuenasTareas ${ }^{\left[{ }^{[10]}\right]}$

Asistimos a transiciones importantes que se expresan como reordenamientos de prácticas letradas que han entrado subrepticiamente en la cultura escolar, creando nuevos mercados y protagonismos en la escena educativa. Varias dinámicas han aportado a esto; por una parte, las normas de edición y autoría se 
vuelven difusas. Cambian las reglas de referenciación, los modelos de consultar; está en curso un proceso de editorialización de la web y de la lectura digital mediante la conjunción de dispositivos técnicos, la fragmentación y recontextualización de contenidos (Cordón, 2016). Para el alumnado, la cuestión les parece sencillamente una ventaja prosaica, pero pragmática, les da oportunidad de salir de sus trabajos con maniobras ramplonas como la de "copiar y pegar" en el procesador de texto.

Estas destrezas utilitarias son muy apreciadas; coincide con que el 61\% de alumnado encuestado en 2014 manifestó la rapidez como la principal ventaja del uso de internet en la elaboración de sus tareas escolares. Esta valoración va de la mano con la aceleración constante de todos los procesos sociales, y lleva la impronta del síndrome de la impaciencia referido por Bauman (2005): refiere al deseo de reducir al máximo el tiempo que separa el deseo con su satisfacción, por lo que cualquier demora se entiende como una evidencia de perjuicio, impotencia e inferioridad en la escala social.

Estos géneros de "consulta" en línea encuentran un terreno fértil para su auge en una cultura escolar laxa y omisa en materia de atención sobre las formas de lectura en soportes digitales y en materia de identificación y valoración de fuentes de información. Estos materiales "listos para usarse” en la realización de tareas escolares resultan funcionales en la medida en que los temas que "desarrollan" corresponden a los contenidos del plan de estudios de un nivel educativo determinado.

Ciertamente, no es que sea una novedad este tipo de recursos para realizar tareas escolares; ya anteriormente circulaban materiales bajo la forma de láminas impresas, ilustradas a color, que se vendían libremente en las papelerías. En la época, se transcribía la información allí contenida; ahora, con la información digitalizada, ya ni siquiera hay necesidad de transcribir el texto. Y entonces como ahora, estas prácticas son posibles en virtud a que en las escuelas no se solicita referenciar los trabajos escolares con un formato preciso, que distinga entre la consulta a la fuente original o en un repositorio como el aquí mencionado: a la pregunta sobre si los profesores solicitan a sus estudiantes referenciar la fuente de información de sus tareas o trabajos escolares, solo el $28 \%$ del alumnado se manifestó afirmativamente; un $48,1 \%$ declara que sus docentes le piden referenciar de manera frecuente; el 23,5\% afirma que casi nunca se le exige. Las formas de citar y referenciar fuentes de información, procesos que fueron clave de la fundamentación en la cultura impresa, no han formado parte constitutiva de la cultura escolar en el bachilleratos y ahora, cuando las formas de apropiación de TIC en las escuelas incluyen la consulta a textos electrónicos que, como nos lo recuerda Chartier (2018), son maleables, susceptibles de ser combinados, recompuestos, y sometidos a una lógica de creación "acumulativa" por parte de quienes utilizan TIC, esta situación tampoco es objeto de análisis ni de intervención con fines de enseñanza y aprendizaje.

\section{Discusión}

En este texto se identificaron un conjunto de prácticas rutinarias que estudiantes realizan con apoyo de TIC para sus tareas escolares, a fin de comprender las relaciones que se establecen con procesos educativos de enseñanza y aprendizaje. Hemos puesto de manifiesto que la integración de TIC en los espacios escolares no ha podido modificar la estructuración de espacios, roles y prácticas, formas de actuación históricamente relacionadas con la cultura impresa.

Mostramos que las escuelas muestran carencias de equipamiento tecnológico, de suficiente, y de un proyecto educativo integral que reconozca y actúe de manera integrada desde los diferentes frentes y actividades que reclaman maneras renovadas d relación con la información y el conocimiento. Se reveló cómo los usos de TIC en las escuelas se ajustan a procesos de enseñanza y aprendizaje tradicionales, y que las instituciones escolares no parecen apremiadas para explorar e innovar con ayuda de estas tecnologías.

La diversificación en los usos de TIC dependerá mayormente del capital cultural de los grupos de jóvenes y no de la educación que desarrollan de las escuelas, que forman escolares con destrezas para una gama de usos reducida e instrumentalista de los recursos tecnológicos, que parecen emplearse para hacer las 
mismas prácticas, pero de manera más eficiente y rápida. No solamente las aulas siguen manteniéndose como sitios para la exposición de contenidos, y las bibliotecas como depósitos de materiales, en un renovado conservadurismo educativo; más preocupante resulta que el uso de TIC por parte de estudiantes parece considerarse en las escuelas como un asunto privado, antes que una cuestión de interés público. Al delegar en cada estudiante la responsabilidad en cuanto a los usos educativos que pueda hacer de estos dispositivos, la utilización de recursos tecnológicos puede convertirse no solo en una expresión más de desigualdad social, sino en su continuación y reforzamiento.

Las formas de apropiación de TIC que se desarrollan en las escuelas no parecen estar guiadas por un examen serio sobre el aprovechamiento educativo de la información accesible en línea, que considere sus cambiantes patrones de estructuración. El enorme universo de información que existe en internet parece percibirse en las escuelas como un raudal sin ordenamiento posible. Se insiste en crear acervos propios de información en línea que no se consultan mediante la creación de bibliotecas virtuales o con el empleo de repositorios de "materiales educativos" relacionados con el plan de estudios del alumnado, lo que denota no solo un deseo pertinaz de mantener un control institucional sobre los textos que se consideran "educativos", sino un extraordinario desconocimiento del régimen actual de producción y circulación de información. Hasta ahora, los parámetros de referenciación de la escritura académica no se han conformado como una cuestión propiamente educativa en las escuelas y no parece entenderse como responsabilidad formativa ni del personal docente, ni del bibliotecario escolar.

\section{Conclusión}

Con la evidencia presentada, puede advertirse que las computadoras no están integradas a procesos de aprendizaje escolar de una forma intencionada y susceptible de evaluación. Algunos datos incluso pueden sugerir que asistimos a una menor capacidad de las escuelas para conducir procesos de enseñanza y aprendizaje cuando se emplean TIC en prácticas escolares. Las formas de apropiación mostradas no parecen ir en la misma dirección de los propósitos educativos de la institución escolar y de las metas que se han trazado y comprometido a alcanzar países enteros en cuanto a promover una educación incluyente, sostenible y sustentable, teniendo a la juventud como principal interlocutora para este empeño.

En este punto, surge una interrogante por el tipo de acciones que son convenientes para tomar el rumbo deseado. Mencionaremos tres claves de respuesta.

En primer término, es evidente la necesidad de cuestionar, de manera decidida, el determinismo tecnológico que existe, no solamente en los discursos sobre el supuesto poder de las TIC en la educación, sino en las acciones y omisiones de las escuelas, que siguen centradas en "ofrecer" equipamiento tecnológico. Conviene también objetar dicho determinismo tecnológico que subyace en los indicadores con que se miden las políticas e inversiones económicas en materia de uso de TIC las escuelas: dotar de equipos no es en sí ningún proyecto propiamente educativo.

Una segunda clave se encuentra en reconocer que la introducción de TIC en las escuelas no puede delimitarse a incorporarlas como una parte más del inventario. Exige un conjunto de reconformaciones en sus formas de trabajo, espacios y funciones educativas. Especial atención merece, en este último rubro, la necesidad de profundizar en los retos pedagógicos que entraña la lectura y gestión de información en soportes digitales.

Por último, resulta imperativo analizar, desde un plano educativo, el entramado de rupturas que las generaciones de jóvenes están realizando con sistemas de valores previos, algunos de los cuales son aún preservados de manera férrea por la escuela tradicional (Smith, Hewitt y Skrbis, 2015), sobre todo los relacionados con la producción de conocimiento, su vigencia y su utilidad. De este modo, se podrá dar una batalla decidida contra las percepciones de insatisfacción, dispersión y superficialidad que estudiantes tienen sobre sus experiencias escolares, como lo registra Linne (2014), situación que explica, al menos en parte, la 
creciente deserción escolar que, en el nivel de educación media superior, alcanza en México una elevadísima tasa de 12,1\% (SEP, 2017). Ahora es el momento de actuar.

\section{REFERENCIAS}

Andrés, G. D. (2014) Una aproximación conceptual a la “apropiación social” de TIC. Questión. Revista especializada en periodismo y comunicación, 1(43), 17-31.

Banco Mundial. (2016) Digital Dividends. World Development Report 2016. Washington D.C.: World Bank. DOI 10.1596/978.1.4648.0728.2

Bauman, Z. (2005). Los retos de la educación en la modernidad líquida. México: Fondo de Cultura Económica.

Boyd, D. (2014). It's complicated. The social lives of networked teens. United Kingdom: Yale University Press.

Cabello, R., López, A. (Eds.). (2017). Contribuciones al estudio de procesos de apropiación de tecnologías. Buenos Aires, Argentina: Ediciones del gato gris/ Red de Investigadores sobre Apropiación de Tecnologías.

Cabero, J., Marín, V. (2014) Educational Possibilities of Social Network and Group Work. University Student's Perceptions. Comunicar, 42(21), 165-172. doi: http://dx.doi.org/10.3916/C42-2014-16

Carr, N. (2011). Superficiales. ¿Qué está haciendo Internet con nuestras mentes? Madrid: Taurus.

Chen, B., Bryer, T. (2012). Investigating Instructional Strategies for Using Social Media in Formal and Informal Learning. The International Review of Research in Open and Distributed Learning, 13(1), 88-104.

Chartier, R. (2018). Libros y lecturas. Los desafíos del mundo digital. Revista de Estudios Sociales 64, 119-124. doi: https://doi.org/10.7440/res64.2018.09.

Claro, M., Espejo, A., Jara, I., Trucco, D. (2011). Aporte del sistema educativo a la reducción de las brechas digitales. Una mirada desde las evaluaciones PISA. Santiago de Chile: Comisión Económica para la América Latina.

Comisión para la Educación de Calidad para Todos. (2016). Construyendo una educación de calidad: Un pacto con el futuro de América Latina. Buenos Aires: Santillana. Recuperado de http://1m1nttzpbhl3wbhhgahbu4ix.wpengine.netdna-cdn.com/wp-content/ uploads/2016/08/Construyendo-una-educacio\%CC\%81n-de-calidad-WEB.pdf

Cordón, J. A. (2016). La lectura en el entorno digital: Nuevas materialidades y prácticas discursivas. Revista Chilena de Literatura 94, 15-38.

Crovi Druetta, D. (2013). Repensar la apropiación desde la cultura digital. En Susana Morales y Ma. Inés Loyola (Comps.), Nuevas perspectivas en los estudios de comunicación. La apropiación tecno-mediática. Buenos Aires: Imago Mundi.

De Certeau, M. (1990) L'invention du quotidien. Vol 1. Arts de faire. Paris: Éditions Gallimard.

Dussel, I. (2010). La escuela y los nuevos medios de información y comunicación. Notas para pensar las relaciones con el saber en la era digital. En Inés Dussel, Viviana Minzi, Fabián Mosesnson, Lila Pagola, Fernando Peirone y Paula Sibilia (Coords.), La educación alterada. Córdoba, Argentina: Salida al Mar / Eduvim.

Ferrés, J., Piscitelli, A. (2012). La competencia mediática: Propuesta articulada de dimensiones e indicadores. Comunicar, 38(19), 75-82. DOI: http//dx.doi.org/10.3916/C38.2012.02.08

García-Gómez, A. (2017). Teen girls and sexual agency: exploring the interpersonal and intergroup dimensions of sexting. Media, Culture \& Society, 39(3), 391-407.

Gomes -Franco, F., Sendín-Gutiérrez, J. C. (2014). Internet como refugio y escudo social: usos problemáticos de la red por jóvenes españoles. Comunicar, 21(43), 45-53. Doi: http://dx.doi.org/10.3916/C43-2014-04

Instituto Nacional para la Evaluación de la Educación (INEE). (2014). Panorama Educativo de México 2013. Educación Básica y Media Superior. Ciudad de México: Autor.

Instituto Nacional para la Evaluación de la Educación INEE). (2017). Panorama Educativo de México. Indicadores del Sistema Educativo Nacional 2016. Educación Básica y Media Superior. Ciudad de México: Autor. 2016. Educación Básica y Media Superior. México: Autor. 
Lankshear, C., y Knobel, M. (2010). Nuevos alfabetismos. Su práctica cotidiana y el aprendizaje en el aula. Madrid: Morata.

Lévy, J. (2010). Actores, sujetos, entornos: Inventar el espacio para leer el mundo. En A. Lindón y D. Hernaux (Eds.), Los giros de la geografía humana (pp. 83-90). México: Anthropos /UAM-I.

Linne, J. (2014). "Ahora hago fast food académico". Los estudiantes de la Universidad de Buenos Aires en tiempos de internet. Palabra clave, 17(3), 695-716. doi: 10.5294/pacla.2014.17.3.7

Manca, S., Ranieritt, M. (2016). Is Facebook still a suitable technology-enhanced learning environment? An updated critical review of the literature from 2012 to 2015. Journal of Computer Assisted Learning, 32(6), 503-528. doi $10.1111 /$ jcal. 12154

Morales,S., Loyola, M. I. (Comps.). (2009). Los jóvenes y las TIC. Apropiación y uso en educación. Córdoba, Argentina: Red de Investigadores sobre Apropiación de Tecnologías.

Mueller, P. A., Oppenheimer, Daniel M. (2014). The Pen Is Mightier Than The Keyboard. Advantages of Longhand Over Laptop Note Taking. Psychological Science, 25(6), 1-10. doi: 10.1177/0956797614524581

OCDE. (2010). Habilidades y competencias del siglo XXI para los aprendices del nuevo milenio en los países de la OCDE. París: Autor.

OCDE. (2012). Connected Minds: Technology and Today's Learners. Paris: Autor. http:// dx.doi.org/10.1787/9789264111011-9-en

OCDE. (2015). Students, Computers and Learning. Making the Connection. http:// dx.doi.org/10.1787/9789264239555-en

Özungur, F. \& Hazer, O. (2018). Analysys of the Acceptance of Communicaction Technologies by Technology Acceptance Model of The Ederly: Example of the Adana Province. International Journal of Eurasia Social Sciences, 9(31), 238-275.

Pantzar, M. (1997). Domestication of Everyday Technology: Dynamics View on the Social History of Artifacts. Design Issues, 13(3), 512-65.

Román, M. y Murillo, J. (2014). Disponibilidad y uso de TIC en escuelas latinoamericanas. Incidencia en el rendimiento escolar. Educação e Pesquisa, 40(4), 879-895. doi :http://dx.doi.org/10.1590/ s1517-97022014121528.

Romero, M., Quesada, A. (2014). Nuevas tecnologías y aprendizaje significativo de las ciencias. Enseñanza de las Ciencias, 32(1), 101-115. doi: http://dx.doi.org/10.5565/rev/ensciencias.433

Sánchez-Rodríguez, J., Ruiz-Palmero, J., Sánchez-Rivas, E. (2015). Uso problemático de las redes sociales en estudiantes universitarios. Revista Complutense De Educación 26, 159-174.

SEP. (2017). Secretaría de Educación Pública. Sistema Nacional de Información Escolar 2015-2016. Disponible en http://www.snie.sep.gob.mx/

Smith, J., Hewitt, B., Skrbis, Z. (2015). Digital socialization: young people's changing value orientations towards internet use between adolescence and early adulthood. Information, Communication and Society, 18(9), 1022-1038. doi: 10.1080/1369118X.2015.1007074

Toboso-Martín, M. (2013). Entre el uso y el no uso de la tecnología: Un enfoque discursivo de la apropiación tecnológica. Intersticios. Revista Sociológica de Pensamiento Crítico, 7(2), 201-212.

Tripp, L. (2011). Digital Youth, Libraries and New Media Literacy. The Reference Librarian, 52, 329-341. doi: $10.1080 / 02763877.2011 .584842$

UNESCO. (2013). Conceptual Relationship of Information Literacy in Knowledge Societies. Paris: Autor.

UNESCO. (2015). Tercer estudio regional comparativo y explicativo. Factores asociados. Santiago de Chile: UNESCO/ Laboratorio Latinoamericano de Evaluación de la calidad de la educación. Recuperado de http:// unesdoc.unesco.org/images/0024/002435/243533s.pdf

UNESCO. (2017). La educación al servicio de los pueblos y el planeta: Creación de futuros sostenibles para todos. Informe de seguimiento de la educación en el mundo. París: Autor.

Van Dijk, J. (2016). La cultura de la conectividad. Una historia crítica de las redes sociales. Buenos Aires: Siglo XXI. 
Weiss, E. (2012). Los estudiantes como jóvenes: El proceso de subjetivación. Perfiles Educativos, 35(135), 134.148.

\section{Notas}

[2]

El acrónimo responde a su nombre en inglés: Programme for International Students Assesment. Es coordinado por la Organización para la Cooperación y el Desarrollo Económicos (OCDE). En México evalúa competencias en lectura -en formato impreso-, matemáticas y ciencias.

[3]

Este estudio se llevó a cabo en 28 países -en Europa, Asia y Oceanía- y tres provincias chinas.

[4]

De acuerdo con los índices del Consejo Nacional de Población (CONAPO) en 2010. Cfr. Índice de Marginación por Localidad 2010. La edición 2010 es la última publicada hasta ahora. www.conapo.gob.mx/es/CONAPO/Indices_de_Marginacion_2010 _municipio

[5]

Nuestra indagación no comprendió las prácticas realizadas en los laboratorios de cómputo, ni aquellas que están directamente enfocadas al aprendizaje de software, a la enseñanza de lenguas extranjeras a través de programas de cómputo especialmente diseñados para tal fin, ni otras prácticas emergentes como la realización de evaluaciones nacionales de desempeño de estudiantes o talleres de robótica. La razón es que estas prácticas no son generalizadas en todas las preparatorias; se realizan solo en un subsistema de bachillerato de los dos que fueron estudiados y exclusivamente en las preparatorias denominadas "metropolitanas" que son las ubicadas en la ciudad capital del Estado de Jalisco, que cuenta con alrededor de cinco millones de habitantes.

[6]

Sobre esta interesante cuestión, Mueller y Oppenheimer (2014) han señalado que la escritura manual tiene mayor efecto en la memoria a largo plazo que si se escribe con una tableta electrónica, debido a los distintos procesos cognoscitivos que implican estos dos tipos de escritura.

[7]

En uno de los sistemas escolares a los que pertenecen unas escuelas de la muestra en estudio se cuenta con una biblioteca virtual, específica para el nivel medio superior, con acceso restringido solo para sus estudiantes.

$[8]$

Datos del Consejo Nacional de Evaluación de la Política de Desarrollo Social http://www.coneval.org.mx/Medicion/ Documents/Pobreza\%202014_CONEVAL_web

En www.buenastareas.com.mx. Consultado en enero de 2014.

En www.buenastareas.com.mx. Consultado en enero de 2017.

\section{BY-NC-ND}

